\title{
Germline mutation of the tumour suppressor PTEN in Proteus syndrome
}

\author{
J M Smith, E P E Kirk, G Theodosopoulos, G M Marshall, J Walker, M Rogers, M Field, \\ J J Brereton, D J Marsh
}

J Med Genet 2002;39:937-940

$\mathrm{P}$ roteus syndrome (PS, OMIM 176920) is a hamartomatous disorder characterised by overgrowth of multiple tissues, connective tissue and epidermal naevi, and vascular malformations. ${ }^{1}$ These presentations are usually apparent at birth or soon after and continue to develop as the patient ages. It is named after the Greek god Proteus who, legend has it, could change his shape at will to avoid capture. It is probably the disease suffered by the Elephant Man. ${ }^{2}$ Tumours, mostly benign but some malignant, have also been reported in PS, generally presenting by the age of 20 years and including papillary adenocarcinoma of the testis, meningioma, and cystadenoma of the ovaries. ${ }^{3}$ Given the predominantly sporadic nature of this syndrome and the mosaic distribution of lesions, it has been suggested that PS may be caused by somatic mosaicism for a genetic change that is lethal in the non-mosaic state.

Clinical overlap, in the form of tissue overgrowth, macrocephaly, and the presence of lipomas, exists between PS and another hamartoma syndrome, Bannayan-Riley-Ruvalcaba syndrome (BRR, OMIM 153480), ${ }^{5}$ in which up to $60 \%$ of affected subjects are known to carry a germline mutation of the tumour suppressor gene PTEN. ${ }^{6}$ BRR also shows partial clinical overlap with Cowden syndrome (CS, OMIM 158350), in which affected subjects are at risk of developing hamartomas in multiple organs including the breast, thyroid, central nervous system, skin, and gastrointestinal tract, as well as malignant tumours of the breast, thyroid, and endometrium. PTEN is mutated in the germline in up to $80 \%$ of patients with CS. ${ }^{6}$ Two recent reports have shown germline, and probably germline mosaic, PTEN mutations in a subset of patients with PS or a PS-like disorder, ${ }^{78}$ although other studies of patients with PS have been unable to confirm these findings. ${ }^{9} 10$

PTEN is a dual specificity phosphatase, shown in vitro to dephosphorylate protein substrates on threonine, serine, and tyrosine residues as well as lipid substrates. ${ }^{6}$ In the phosphatidylinositol 3-kinase (PI3-kinase) pathway, PTEN functions as a 3 phosphatase, dephosphorylating phosphatidylinositol $(3,4,5)$ triphosphate $\left[\operatorname{Ptd} \operatorname{Ins}(3,4,5) \mathrm{P}_{3}\right]$ to PtdIns $(4,5) \mathrm{P}_{2}$. When PTEN is mutant, PtdIns $(3,4,5) \mathrm{P}_{3}$ accumulates, activating the serine threonine kinase AKT, a known anti-apoptotic factor, leading to cell survival. In addition to being mutated in the germline in CS and BRR, somatic loss of function PTEN mutations are seen in a wide range of sporadic human tumours, including endometrial cancer, glioblastoma, and prostate cancer. Given the clinical overlap between PTEN associated syndromes and PS, as well as the conflicting studies of PTEN and PS recently published, we sought to determine whether a patient presenting with classical PS harboured a germline PTEN mutation.

\section{MATERIALS AND METHODS}

\section{Case report}

The patient, now aged 16 months, is the second child of nonconsanguineous parents. He was born by normal vaginal

\section{Key points}

- Proteus syndrome (PS) is a hamartomatous disorder characterised by overgrowth of multiple tissues that usually manifests early and continues to develop as the patient ages. Clinical overlap exists between PS and another hamartoma syndrome, Bannayan-RileyRuvalcaba (BRR) syndrome.

- Up to $60 \%$ of subjects affected by BRR carry a germline mutation of the tumour suppressor gene PTEN, which encodes a dual specificity phosphatase. PTEN mutations can also be identified in the germline of up to $80 \%$ of subjects with Cowden syndrome (CS), characterised by the presence of hamartomas in multiple organs as well as breast, thyroid, and endometrial malignancies.

- We report a 16 month old male with a de novo PTEN mutation, c.507delC, and classical features of PS, including a left sided epidermal naevus following the lines of Blaschko, widespread capillary venous malformation on his chest and abdomen, multiple lipoblastomata, disproportionate overgrowth of the right leg, and a progressive course.

- This mutation is predicted to prematurely truncate the $\mathrm{COOH}$-terminal C2 domain of PTEN, losing regions critical for PTEN stability and protein-protein interactions. The finding of a de novo PTEN mutation in this patient supports the previous finding of PTEN mutations in two cases of PS and four cases of patients with a PS-like disorder.

- Thus, the molecular diagnosis of a germline PTEN mutation classifies these PS cases, along with CS and BRR, as part of the PTEN hamartoma syndrome (PHTS) spectrum. These findings have implications for cancer risk and future clinical surveillance in patients with PS.

delivery at 38 weeks of gestation after an uncomplicated pregnancy. His birth weight was $3735 \mathrm{~g}$ (>90th centile), length was $52.5 \mathrm{~cm}$ (>90th centile), and head circumference was $47.6 \mathrm{~cm}$ (>90th centile). His 3 year old sister is well. The proband's mother presented at 33 years with abdominal pain after meals and occult blood in her stool. Endoscopy showed multiple fundic glandular polyps in the stomach and two small, hyperplastic distal rectal polyps. The proband's maternal grandmother and great grandmother developed unilateral breast cancer aged 60 and 48 respectively. In addition, five great aunts of the proband, from different sides of the maternal grandparents' family, developed unilateral breast cancer in their seventies. Given the relatively late age of onset of breast cancer in these people, its unilateral nature, and its presence in unrelated subjects in this pedigree, family members have not been entered into a BRCAI or BRCA2 genetic testing 

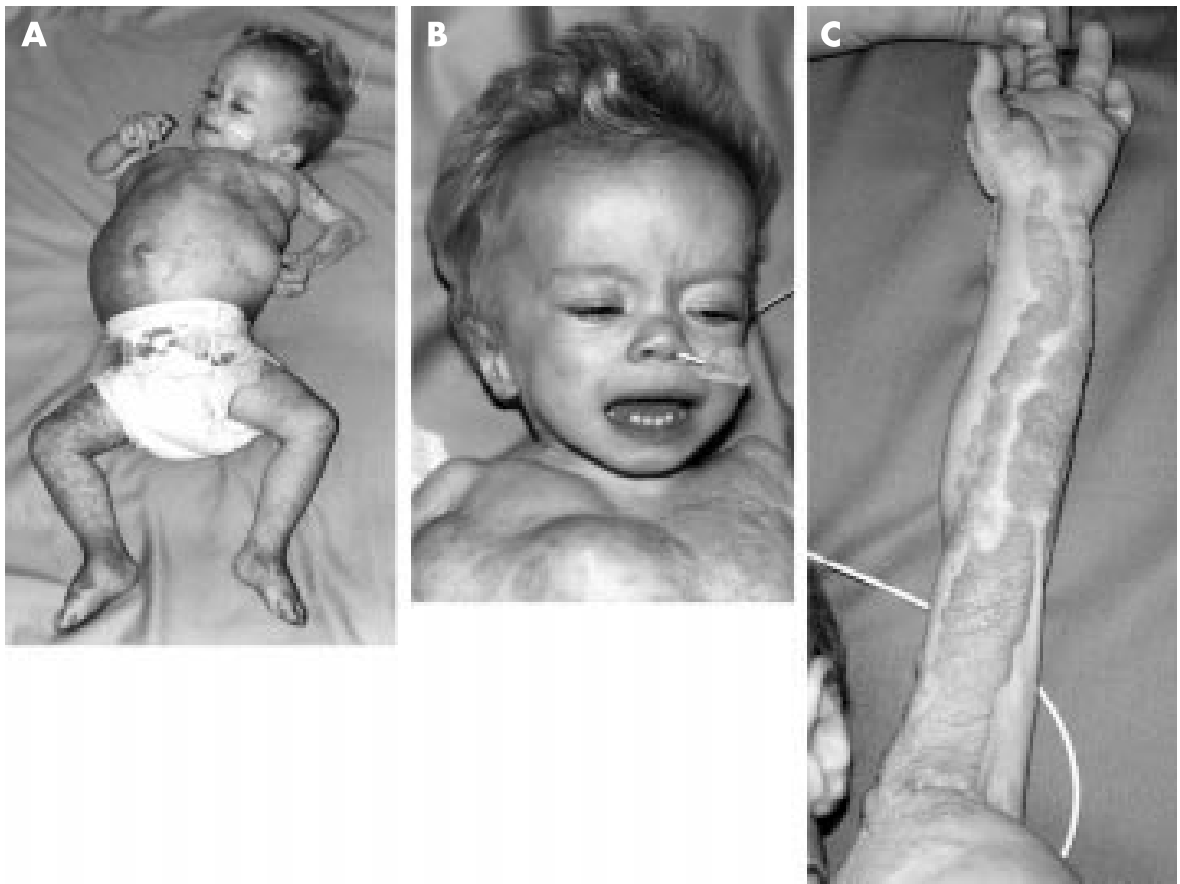

Figure 1 The proband at 15 months. (A) Full body view. Note asymmetry of lower limbs, vascular malformation mainly visible on the right side, epidermal naevus on the left, and lipomas on the trunk. The limbs (other than the right leg) are wasted. (B) Front view of the proband's face, showing prominent forehead and flattened nasal bridge. Lipomas are visible on the upper chest. (C) Left arm showing epidermal naevus conforming to the lines of Blaschko.

programme. There is no family history of thyroid carcinoma or additional stigmata, such as macrocephaly or trichilemmomas in either the proband's mother or her relatives, as far as can be assessed, which may be consistent with a diagnosis of CS.

At birth, there was an extensive epidermal naevus on the left side of the proband's body following the lines of Blaschko involving his left arm, hand, chest, and flank. There was also a widespread capillary venous malformation on his chest, abdomen, and right leg. There were two small palpable lesions on the right side of his chest that enlarged over the following months. A biopsy at 4 months of age of two separate lesions showed a combination of immature and mature fat cells consistent with lipoblastomatosis in one and a vascular malformation in the other. Over time he has developed multiple lipomata on his trunk which have continued to enlarge, particularly those on the thorax. At 12 months of age there was evidence of measurable, disproportionate overgrowth of the right leg. A skeletal survey at 11 months of age was normal. The capillary venous malformation and epidermal naevus have remained unchanged (fig 1 ).

From 5 to 8 months of age he had multiple admissions with respiratory illnesses. A CT (computerised tomography) scan of his chest and abdomen at 7 months of age showed a large lipoma of the right lateral chest wall and a smaller lipomatous lesion of the left lateral and anterior chest wall. There was no extension into the chest or abdomen. A subsequent CT chest scan performed because of persistently increased effort of breathing at 10 months showed a large anterior mediastinal mass and extensive mesenteric lymphadenopathy. There was no visceral or intracranial vascular malformation. A CT guided fine needle biopsy of the mediastinal mass showed lipoma. Laryngoscopy, bronchoscopy, and oesphagoscopy showed extrinsic compression of the left bronchus presumed secondary to the mediastinal mass. Biopsies of lesions on the right side of the thorax showed lipomata and a venous haemangioma. Endoscopy and colonoscopy performed at 10 months of age because of lower gastrointestinal bleeding and iron deficiency anaemia showed multiple sessile polypoid lesions in the jejunum and the colon. Several of the colonic polyps were biopsied and on histopathology the appearance was of large bowel mucosa with prominent mucosal lymphoid tissue but no other abnormalities. Upper gastrointestinal biopsies showed mild reflux oesphagitis and gastritis only.

He has linear growth failure, crossing from the 50th centile at 10 months $(73.5 \mathrm{~cm})$ to between the 1st and 3rd centiles at 15 months $(74.5 \mathrm{~cm})$. During the same period, despite strikingly decreased muscle bulk and subcutaneous fat, his weight centile has increased from the 25th to the 50th centile, possibly owing to the rapid growth of his lipomas. Enteral feeds were started at 9 months of age. His head circumference was $48 \mathrm{~cm}$ (50th to 98 th centile) at 15 months. He has a prominent forehead and a flat nasal bridge. Intellectually he appears to be within normal limits, although his physical condition limits his abilities. Routine karyotyping of blood lymphocytes and of skin fibroblasts from a region of skin affected by superficial vascular lesions showed a normal male karyotype, 46,XY.

\section{Molecular analysis}

PTEN mutation scanning was undertaken using DNA extracted from peripheral blood collected from the proband and his parents as well as a fibroblast cell culture established from a skin biopsy of an area affected by superficial vascular lesions. DNA was extracted using the Puregene ${ }^{\mathrm{TM}}$ DNA Purification System (Gentra Systems, Minneapolis, MI, USA). All patients, or guardians thereof, signed informed consent for genetic testing as approved by the NSW Genetics Service.

PTEN sequencing primers have been previously described. ${ }^{11-13}$ PTEN exons and flanking intronic sequences were amplified using Platinum ${ }^{\circledR}$ Taq DNA polymerase and the accompanying standard $10 \times$ PCR buffer (Invitrogen, Adelaide, SA, Australia) with the exception of exon 1 that required the $10 \times$ PCRx Amplification Buffer from the PCRx Enhancer System (Invitrogen, Adelaide, SA, Australia). PCR reaction conditions were as follows: $94^{\circ} \mathrm{C}$ annealing for five minutes; 40 cycles of $94^{\circ} \mathrm{C}$ for one minute, $55^{\circ} \mathrm{C}$ for one 

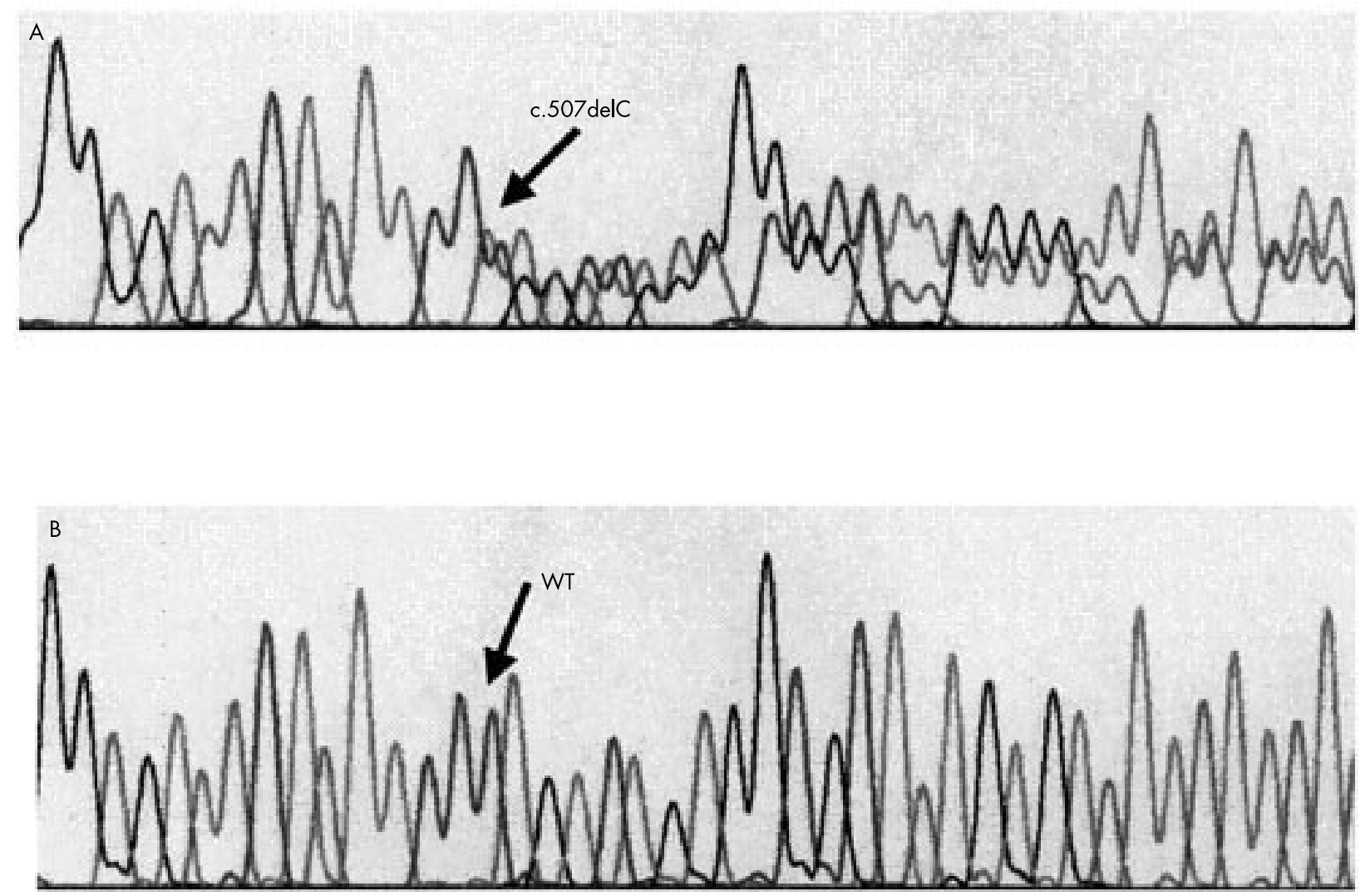

Figure 2 Sequence of PTEN exon 6. (A) c.507delC in the proband's constitutive DNA. (B) Wild type constitutive DNA.

minute, and $72^{\circ} \mathrm{C}$ for one minute; followed by $72^{\circ} \mathrm{C}$ for 10 minutes. PCR products were purified using the Wizard PCR Preps DNA Purification System (Promega, Madison, WI, USA). Direct sequencing of purified PCR products was performed using the ABI Prism BigDye Terminator cycle sequencing ready reaction kit (Applied Biosystems, PerkinElmer Corp, USA). Purified cycle sequencing products were electrophoresed on 4.8\% PAGE Plus gels (Amresco, Cleveland, $\mathrm{OH}$, USA) and analysed on a 377XL automated DNA sequencer (Applied Biosystems, Perkin-Elmer Corp, USA) at the Australian Genome Research Facility (University of Queensland, St Lucia, QLD, Australia).

\section{RESULTS}

Mutation scanning of the entire coding and flanking intronic sequence of PTEN showed a de novo germline mutation in exon 6, c.507delC, in the constitutive DNA of the proband with PS but not in either of his parents (fig 2). This mutation is predicted to introduce a stop codon (TAA) 38 nucleotides downstream within exon 6 that would probably lead to premature truncation of the PTEN protein. To our knowledge, c.507delC has not been reported to be associated with either CS or BRR, nor is it reported in the Human Gene Mutation Database (http://archive.uwcm.ac.uk/uwcm/mg/hgmdo. html). PTEN scanning of the DNA extracted from a fibroblast cell line established from a skin biopsy of a region of superficial vascular lesions did not show any additional mutations that may have occurred at the somatic level in these cells or reflect germline mosaicism for PTEN. Further, both the wild type PTEN allele and the mutant allele were retained in these cells.

\section{DISCUSSION}

Difficulty in the diagnosis of PS because of both its variability of expression and clinical overlap with other conditions, including the hamartoma syndrome BRR, is well recognised. Until recently, the genetic events involved in the development of PS that may assist by providing a "molecular diagnosis" have not been understood. Two reports have, in total, described germline PTEN mutations in two of $14(14 \%)$ patients with PS and four of six (67\%) patients with a PS-like disorder. ${ }^{78}$ We have confirmed the presence of germline PTEN mutation in a subset of patients with PS by the identification of a de novo PTEN mutation in a patient with classical PS conforming to the criteria described by Biesecker et al. ${ }^{1}$ Specifically, our patient meets the mandatory general criteria for PS of mosaic distribution of lesions, a rapidly progressive clinical course, and sporadic presentation. We have confirmed the sporadic presentation at the molecular level, by the identification of a germline PTEN mutation subsequently shown to be absent in the proband's parents. No additional mutation in the PTEN coding and flanking intronic sequence was identified in this patient. In addition, positive findings in the specific criteria for the diagnosis of PS include category B, two signs (epidermal naevus and disproportionate overgrowth) and category $\mathrm{C}$, two signs (evidence of dysregulated adipose tissue, with both lipomas and regional absence of fat, and venous malformation).

With the exception of R335X (exon 8) that has been identified in germline DNA from two patients with a PS-like disorder and also in CS and BRR/CS overlap families, ${ }^{14}$ the PTEN mutations reported in PS and PS-like disease, M35T (exon 2), W111R (exon 5), W211X (exon 6), and the mutation reported in the current study, c.507delC (exon 6), have not been previously associated with CS or BRR. ${ }^{78}$ Further, Zhou et al $7^{7}$ identified a second "hit" in PTEN in the form of the Rl30X mutation in three physically non-contiguous sites, specifically a lipomatous mass, an epidermoid naevus, and in arteriovenous malformation tissue in a patient with PS-like disorder with the R335X mutation. This mutation occurred on the opposite 
allele to $\mathrm{R} 335 \mathrm{X}$, probably representing germline mosaicism and possibly explaining the mosaic distribution of lesions seen in this PS patient by complete inactivation of PTEN. R130X, located in the interval encoding the PTPase core motif of PTEN, is a relatively frequent mutation, seen in CS, BRR, and CS/BRR overlap families. ${ }^{14}$

We did not identify a second mutation in PTEN in DNA extracted from a skin fibroblast culture of a region of superficial vascular lesions from our proband and were able to show retention of heterozygosity at the PTEN locus in this tissue. In this way, our data are consistent with the report of five cases of PTEN mutation associated with PS or PS-like disease. ${ }^{8}$ Like the previously reported nonsense mutations in exons 6 and 8 of PTEN, c.507delC is predicted to truncate prematurely the COOH-terminal C2 domain that has been shown to be important for phospholipid membrane binding. ${ }^{15}$ This loss encompasses a PDZ-binding motif encoded by the last four amino acids of PTEN (ITKV) known to be important for proteinprotein interactions, as well as PEST sequences critical for PTEN stability. ${ }^{16}$

It is possible that PTEN may be inactivated in affected tissue by epigenetic events such as methylation or, alternatively, that haploinsufficiency of PTEN was sufficient to cause much of the phenotype observed in these patients, as has been shown in tumours from one of the pten knock out mouse models, pten $+/-{ }^{17}$ However, there is clear evidence for mosaicism in this patient, as in many others with PS. Specifically, his epidermal naevus clearly follows the lines of Blaschko. Given the lack of evidence for a second PTEN mutation in this and other similar children with PS, the likelihood that a second genetic event occurs in a gene other than PTEN cannot be excluded. At least in some patients, PS may be a digenic disorder.

The child described in this study has classical features diagnostic of PS, thus confirming the findings of Zhou et al that PTEN mutations are found in a subset of patients with PS. Clearly, however, PS is a genetically heterogeneous condition and additional underlying genetic causes of this syndrome account for the majority of PS cases and remain to be identified. A total of 41 patients with either PS $(n=39)$ or PS-like disorder $(\mathrm{n}=2)$ have been reported who do not have a germline PTEN mutation. ${ }^{7-10}$ Including the child described in this paper, a total of three patients with PS ( 3 of $41,7 \%$ ) and four patients with PS-like disease (4 of 6, 67\%) have now been reported to have germline mutation of PTEN. The molecular diagnosis of PTEN mutation positive classifies these PS patients, along with CS and BRR, as part of the PTEN hamartoma syndrome (PHTS) spectrum. As such, patients surviving the early years of PS will likely be at a greater risk of developing thyroid, breast, and endometrial malignancies as they age and clinical surveillance for these tumours should be considered.

\section{ACKNOWLEDGEMENTS}

We would like to thank the family of the proband for their help during the preparation of this paper. DJM is a R D Wright Fellow (NHMRC, Australia). Roberto Zori is thanked for helpful discussions. Bruce Robinson is acknowledged for his support.

\section{Authors' affiliations}

J M Smith, E P E Kirk, M Field, Department of Medical Genetics, Sydney Children's Hospital, High Street, Randwick, NSW 2031,

Australia
G Theodosopoulos, D J Marsh, Kolling Institute of Medical Research, Royal North Shore Hospital, and Department of Molecular Medicine, University of Sydney, St Leonards, NSW 2065, Australia

G M Marshall, Centre for Children's Cancer and Blood Disorders, Sydney Children's Hospital, High Street, Randwick, NSW 2031, Australia

J Walker, Department of Endocrinology, Sydney Children's Hospital, High Street, Randwick, NSW 2031, Australia

M Rogers, Department of Dermatology, The Children's Hospital at Westmead, Westmead, NSW 2145, Australia

J J Brereton, Visiting Medical Officer, Wollongong Hospital, Crown Street, Wollongong, NSW 2500, Australia

Correspondence to: Dr D J Marsh, Kolling Institute of Medical Research, Royal North Shore Hospital, St Leonards, NSW 2065, Australia: Debbie Marsh@med.usyd.edu.au or Dr E P E Kirk, Department of Medical Genetics, Sydney Children's Hospital, High Street, Randwick, NSW 2031, Australia; KirkED@sesahs.nsw.gov.au

\section{REFERENCES}

1 Biesecker LG, Happle R, Mulliken JB, Weksberg R, Graham JM, Viljoen $\mathrm{DL}$, Cohen MM. Proteus syndrome: diagnostic criteria, differential diagnosis, and patient evaluation. Am J Med Genet 1999;84:389-95.

2 Cohen MM. Invited historical comment. Further diagnostic thoughts about the elephant man. Am J Med Genet 1988;29:777-82.

3 Gordon PL, Wilroy RS, Lasater OE, Cohen MM. Neoplasms in Proteus syndrome. Am J Med Genet 1995;57:74-8.

4 Cohen MM. Proteus syndrome: clinical evidence for somatic mosaicism and selective review. Am J Med Genet 1993;47:645-52.

5 Bialer MG, Riedy M, Wilson WG. Proteus syndrome versus Bannayan-Zonana syndrome: a problem in differential diagnosis. Eur J Pediatr 1988;148:122-5.

6 Waite KA, Eng C. Protean PTEN: form and function. Am J Hum Genet 2002; 70:829-44

7 Zhou XP, Marsh DJ, Hampel H, Mulliken JB, Gimm O, Eng C. Germline and germline mosaic PTEN mutations associated with a Proteus-like syndrome of hemihypertrophy, lower limb asymmetry, arteriovenous malformations and lipomatosis. Hum Mol Genet 2000; 9:765-8

8 Zhou XP, Hampel H, Thiele H, Gorlin RJ, Hennekam RCM, Parisi M, Winter RM, Eng C. Association of a germline mutation in the PTEN tumor suppressor gene and Proteus and Proteus-like syndromes. Lancet 2001;358:210-11.

9 Barker K, Martinez A, Wang R, Bevan S, Murday V, Shipley J, Houlston $R$, Harper J. PTEN mutations are uncommon in Proteus syndrome. J Med Genet 2001;38:480-1.

10 Biesecker LG, Rosenberg M, Vacha S, Turner JT, Cohen MM. PTEN mutations and Proteus syndrome. Lancet 2001;358:2079.

11 Liaw D, Marsh DJ, Li J, Dahia PLM, Wang SI, Zheng Z, Bose S, Call KM, Tsou HC, Peacocke M, Eng C, Parsons R. Germline mutations of the PTEN gene in Cowden disease, an inherited breast and thyroid cancer syndrome. Nat Genet 1997:16:64-7.

12 Steck PA, Pershouse MA, Jasser SA, Yung WKA, Lin $\mathrm{H}$, Ligon AH, Langford LA, Baumgard ML, Hattier T, Davis T, Frye C, Hu R, Swedlund $B$, Teng DHF, Tavitigian SV. Identification of a candidate tumour suppressor gene, $M M A C l$, at chromosome 10q23.3 that is mutated in multiple advanced cancers. Nat Genet 1997:15:356-62.

13 Marsh DJ, Dahia PLM, Coulon V, Zheng Z, Dorion-Bonnet F, Call KM, Little R, Lin AY, Eeles RA, Goldstein AM, Hodgson SV, Richardson AL, Robinson BG, Weber HC, Longy M, Eng C. Allelic imbalance, including deletion of PTEN/MMACl, at the Cowden disease locus on 10q22-23, in hamartomas from patients with Cowden syndrome and germline PTEN mutation. Genes Chrom Cancer 1998;21:61-9.

14 Marsh DJ, Kum JB, Lunetta KL, Bennet M, Gorlin RJ, Ahmed F, Bodurtha J, Crowe C, Curtis MA, Dasouki M, Dunn T, Feit H, Geraghty MT, Graham JM, Hodgson SV, Hunter A, Korf BR, Manchester D, Miesfeldt S, Murday VA, Nathanson KL, Parisi M, Pober B, Romano C, Tolmie JL, Trembath R, Winter RM, Zackai EH, Zori RT, Weng LP, Dahia PLM, Eng C. PTEN mutation spectrum and genotype-phenotype correlations in Bannayan-Riley-Ruvalcaba syndrome suggest a single entity with Cowden syndrome. Hum Mol Genet 1999;8:1461-72.

15 Georgescu MM, Kirsch KH, Kaloudis P, Yang HJ, Pavletich NP Hanafusa $\mathrm{H}$. Stabilization and productive positioning roles of the $\mathrm{C} 2$ domain of PTEN tumor suppressor. Cancer Res 2000;60:7033-8.

16 Lee JO, Yang H, Georgescu MM, Cristofano AD, Maehama T, Shi Y, Dixon JE, Pandolfi P, Pavletich NP. Crystal structure of the PTEN tumour suppressor: Implications for its phosphoinositide phosphatase activity and membrane association. Cell 1999;99:323-34.

17 Di Cristofano A, Pesce B, Cordon-Cardo C, Pandolfi PP. Pten is essential for embryonic development and tumour suppression. Nat Genet 1998; 19:348-55. 